\title{
A Monte Carlo study of the Falicov-Kimball model in the perturbative regime
}

\author{
Grzegorz Musiał ${ }^{1}$, Lech Dębski ${ }^{1}$, and Jacek Wojtkiewicz ${ }^{2}$ \\ ${ }^{1}$ Institute of Physics, Adam Mickiewicz University, 85 Umultowska Ul., Poznań 61-614, Poland \\ E-mail: gmusial@amu.edu.pl; gulf@spin.amu.edu.pl \\ ${ }^{2}$ Dept. for Math. Methods in Physics, Warsaw University, 74 Hoża Ul., Warszawa 00-682, Poland \\ E-mail: wjacek@fuw.edu.pl
}

Received January 18, 2007, revised April 2, 2007

\begin{abstract}
Finite-temperature properties of the Falicov-Kimball model on the square lattice have been studied in the perturbative regime, i.e. for $t / U<<1$, where $t$ is the hopping constant and $U$ denotes the Coulomb interaction strength. In our study, we have determined the phase diagram of the model in the second-order of the perturbation theory, where the antiferromagnetic Ising model in the magnetic field emerges. In the fourth-order, where our model constitutes the Ising model with more complicated frustrated antiferromagnetic interactions, the sketch of the phase diagram was established. The Monte Carlo method was employed and the behavior of Binder cumulants based on the order parameters was analyzed to determine the type of ordering and phase boundaries in the diagram.
\end{abstract}

PACS: 71.10. Hf Non-Fermi-liquid ground states, electron phase diagrams and phase transitions in model systems;

75.10.Hk Classical spin models;

75.30.Kz Magnetic phase boundaries;

75.40.Mg Numerical simulation studies.

Keywords: Monte Carlo simulations, 2D Falicov-Kimball model, perturbation theory.

\section{Introduction}

The Falicov-Kimball model (FKM) [1] is one of simplest possible lattice model of itinerant interacting fermions. In this model, two sorts of particles are present: classical («heavy») particles described by occupation number $w_{x}$, which can take two values: 0 and 1 , and quantum itinerant spinless fermions, described by creation and annihilation operators $c_{x}^{\dagger}, c_{x}$. The Hamiltonian, defined on the subset $\Lambda$ of the lattice, is

$$
=-\sum_{x, y \in \Lambda} t_{x y} c_{x}^{\dagger} c_{y}+U \sum_{x \in \Lambda}^{H_{\Lambda}\left(\left\{w_{x}\right\}\right)=} w_{x} n_{x}+\sum_{x \in \Lambda} \mu_{+} w_{x}+\sum_{x \in \Lambda} \mu_{x} c_{x}^{\dagger} c_{x} .
$$

In the formula above, $t_{x y}$ are hopping constants and we assume here that they are equal to $t$, if $x, y$ are nearest-neighbors (nn) and zero otherwise. $\mu_{+}, \mu_{-}$are chemical potentials for heavy and itinerant particles, respectively.

This model has been used to describe numerous phenomena in solid state physics: semiconductor-metal tran- sitions, appearance of ordering(s) in mixed-valence compounds and binary alloys, non-resonant Raman scattering (for exhaustive review, see [2]).

Moreover, the behavior of the FKM can serve as a guide and a source of hints in investigation of more general models, such as the Hubbard model, where exact results still are rare. On heuristic grounds one can expect that there are interrelations between certain phenomena in both models (for instance, the segregation observed in FKM would correspond to ferromagnetism in the Hubbard model [3]).

There exist numerous both rigorous and numerical results on the area of the FK model. Most of them concern orderings in ground states and in low temperatures (for representative sample of rigorous results see [4]). However, temperature-driven phase transitions are much less known.

This opportunity motivated us to investigate this problem. We begin the study from the simplest non-trivial case, i.e. two-dimensional model at half-filling in the perturbative regime (i.e., when the hopping constant $t$ is 
much smaller than the strength of Coulomb interaction $U)$. In this range of parameters one can use perturbation expansion and the FKM is equivalent to the Ising-like model. In the second order of perturbation theory, one obtains the antiferromagnetic nn Ising model with the Hamiltonian:

$$
H_{\mathrm{eff}}^{(2)}=h \sum_{i} s_{i}+2 \frac{t^{2}}{U} \sum_{d(i, j)=1} s_{i} s_{j}
$$

( $s_{i}$ in the formula above is related to $w_{i}$ by: $s_{i}=w_{i}-1 / 2$ ). In the fourth order one obtains the Ising model with complicated frustrated short range (up to two lattice spacings) interactions:

$$
\begin{aligned}
H_{\mathrm{eff}}^{(4)} & =h \sum_{i} s_{i}+\left(2 \frac{t^{2}}{U}-18 \frac{t^{4}}{U^{3}}\right) \sum_{d(i, j)=1} s_{i} s_{j}+\frac{6 t^{4}}{U^{3}} \sum_{d(i, j)=\sqrt{2}} s_{i} s_{j}+ \\
& +\frac{4 t^{4}}{U^{3}} \sum_{d(i, j)=2} s_{i} s_{j}+\frac{40 t^{4}}{U^{3}} \sum_{\mathbb{P}_{4, i j k l}} s_{i} s_{j} s_{k} s_{l}+\frac{3 t^{4}}{2 U^{3}} \sum_{\mathbb{P}_{4, i j k l}} \mathbf{1}
\end{aligned}
$$

$\left(\mathscr{P}_{4, i j k l}\right.$ is a unit square plaquette spanned by sites $\left.i, j, k, l\right)$. We study this effective model with the use of Monte Carlo method - perhaps the most universal method in the area of spin systems. Ground state orderings [5] presented in Fig. 1 are our reference point.

It is worth noting that one can apply the quantum Monte Carlo method to the FKM, as the famous minus-sign problem for fermions does not appear here. This method is applicable for arbitrary filling (no limitation to half-filled case) and arbitrary value of $t / U$. The price paid is however the amount of computer time (2-4 orders of magnitude larger to obtain the precision comparable to our approach) [6].

\section{The Monte Carlo simulations}

The MC simulations presented here are adapted from the simulations presented in [7]. We have generated equilibrium configurations of the finite-size square spin samples of the size $L \times L(L \leq 54)$ for fixed values of the model parameters, using the Metropolis algorithm. Periodic

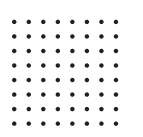

$a$

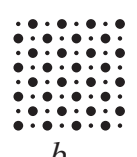

$b$

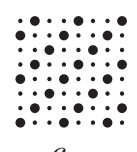

c

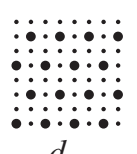

$d$

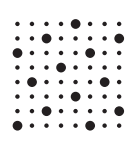

e
Fig. 1. The ground state orderings for effective Hamiltonian (3) in the fourth order of the perturbation theory. The configurations of the heavy particles (marked by bold dots $(\bullet)$ ) in parts $(a),(b),(c),(d)$ and $(e)$ correspond to the phases referred as (0), (1), (2), (3) and (4), respectively. boundary conditions were imposed and thermalization of the initial configurations of the length of $10^{5}$ to $10^{6}$ Monte Carlo steps (MCS) was applied, depending on the size of a sample. The 48-bit random number generator was used. Each MC run was split into $k(6 \leq k \leq 40)$ segments consisting of $10^{7} \mathrm{MCS}$ to calculate partial averages. In the calculation of the partial averages only every $i$ th $\mathrm{MC}$ step contributes $(6 \leq i \leq 10)$, to avoid correlations between sampled configurations of spins in the system.

To find the phase transition points, the Binder cumulant [7] $Q_{L}=\left\langle M_{\alpha}^{2}\right\rangle_{L}^{2} /\left\langle M_{\alpha}^{4}\right\rangle_{L}$ was used. Here $\left\langle M_{\alpha}^{n}\right\rangle_{L}$ denotes the $n$th power of the $\alpha$ spins order parameter, averaged over an assembly of independent samples of the size $L \times L$. For $T>T_{c}$ and $L>>\xi$, where $\xi$ denotes the correlation length, $Q_{L}$ tends towards $1 / 3$ which corresponds to a Gaussian distribution, whereas for $T<T_{c}$ and $L>>\xi, Q_{L}$ tends to 1 . For $L<<\xi$, the common intersection point of the curves $Q_{L}$ appears, which should be identified with the phase transition point. This analysis we call the intersection method.

Assuming $t / U=1 / 10$ and applying this method, we obtained the phase diagram of the model with Hamiltonian (2) (i.e., in the second order of the perturbation theory) which is presented in Fig. 2. Together with the ground state orderings [5] (Fig. 1), it makes the reference point for investigation of the model with Hamiltonian (3) (i.e., in the fourth order of the perturbation theory). In our study of the latter model, we started from the calculation of the phase transition point at $h=0$. Using the intersection method, we obtained the critical value $k_{B T}=$ $=0.3280(2)$, marked with $(x)$ in Fig. 3. To determine the way of ordering in the system, we investigated the behavior of the Binder cumulant with the use of each of the two alternative sublattices (see Fig. 1,b), which implied the

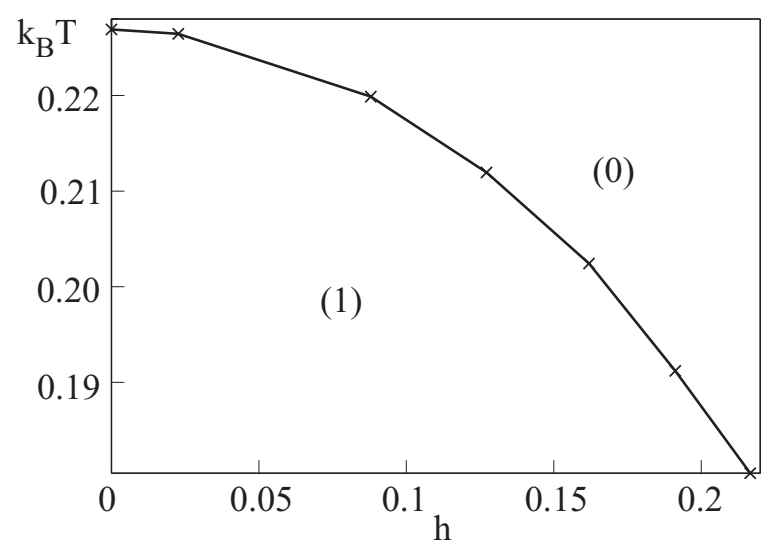

Fig. 2. The phase diagram of the 2D Falicov-Kimball model on a square lattice in the second order of perturbation theory. $h$ denotes the difference of the chemical potentials $\mu_{+}$and $\mu_{-}$ for heavy and itinerant particles, respectively. The points, for which the calculations were performed within this paper, are marked with $(x)$ and the lines are drawn to guide eyes. Labels of phases are explained in the caption of Fig. 1. 


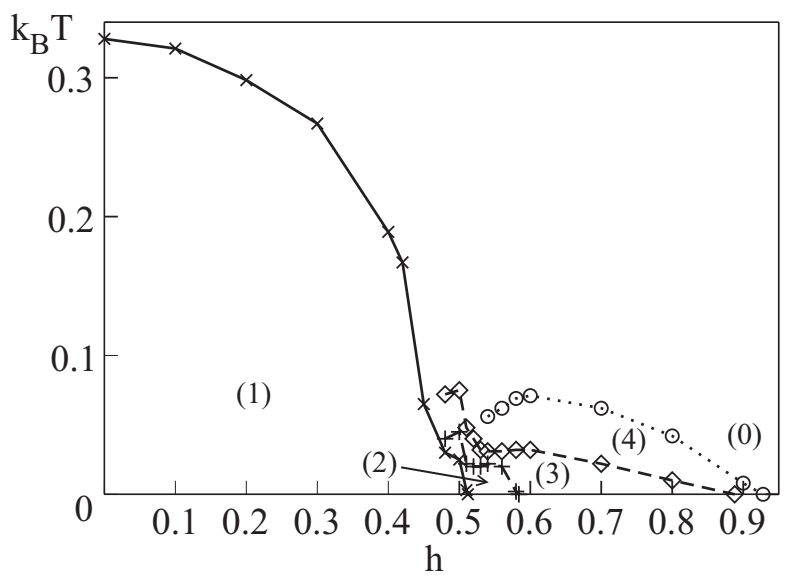

Fig. 3. The sketch of the phase diagram of the 2D Falicov-Kimball model on a square lattice in the fourth order of perturbation theory. The points, for which the calculations were performed within this paper, are marked with symbols and the lines are drawn to guide eyes. Labels of phases are explained in the caption of Fig. 1.

presence of phase (1). This fact was confirmed by the behavior of the cumulant $Q$ determined with the use of each of the three alternative sublattices (see Fig. 1,c). We observed only the parallel horizontal curves for each value of the system size $L$. Analogously we determined the critical values of $k_{B} T$ for the phase transitions at $h=0.1$ to 0.45 . The behavior of the cumulant $Q$ with the use of each of the three alternative sublattices gave no evidence of phase (2) illustrated in Fig. 1,c.

Only for $h \geq 0.4 \geq 0.45$ in the phase transition region we observed small oscillations of the values of the Binder cumulants having divided the lattice into two sublattices or into three sublattices, signalizing an increase in the contribution of the further terms in Hamiltonian (3) to the interaction energy of degrees of freedom, although in the latter case the lines for different $L$ 's still did not intersect, which ment that phase (2) was not realized.

We univocally detected phase (2) and the next ones presented in Fig. 1 only in the simulations for $h \geq 0.48$. Figs. 4 and 5 show the exemplary $k_{B} T$ dependence of the Binder cumulants $Q$ at $h=0.5$. Having in mind the above mentioned properties of the Binder cumulant, we can conclude that for $k_{B} T \leq 0.025$ phase (1) appears. It follows from the dependencies $Q\left(k_{B} T\right)$ for various $L$, with the use of each of the two alternative sublattices, as illustrated in Fig. 4. The increase in the values of $Q$ and the appearance of intersections of the dependences $Q\left(k_{B} T\right)$ for $L=39$ and 51 (odd values, which are divisible by 3 , because of the periodic boundary conditions), illustrated in Fig. 5, show that phase (2) appears whose upper boundary point can be estimated as about $k_{B} T=0.045$. For higher values of $k_{B} T$ between 0.045 and 0.075 phase (3) appears. Its appearance is reflected by the increase in values of $Q$ presented in

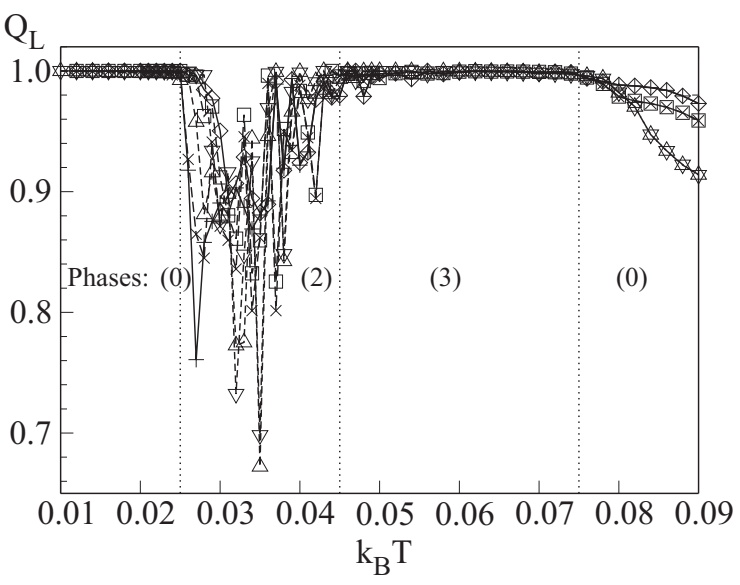

Fig. 4. The $k_{B} T$ dependence of two Binder cumulants $Q$ calculated for the samples with different linear size $L$, listed in the legend box, at $h=0.5$. Each cumulant $Q$ was calculated from the one of two sublattices shown in Fig. 1,b. Phase (1) is absent here. $L=30$, sublattice A $(\nabla) ; L=30$, sublattice B $(\triangle)$; $L=42$, sublattice A $(\square) ; L=42$, sublattice B $(\times) ; L=54$, sublattice A $(\diamond) ; L=54$, sublattice B $(+)$.

Fig. 4. This region ends at $k_{B} T=0.075$ above which the disordered phase ( 0 ) appears. It is evident that the appearance of phases (3) and (4) influences the behavior of the cumulants explained in the captions of Figs. 4 and 5 which allowed us to estimate the boundaries of these phases. Strict investigation of these phases needs a construction of other Binder cumulants for more complicated situation illustrated in Figs. $1, d$ and 1,e, but the computer program and the analysis of the results would be very complex.

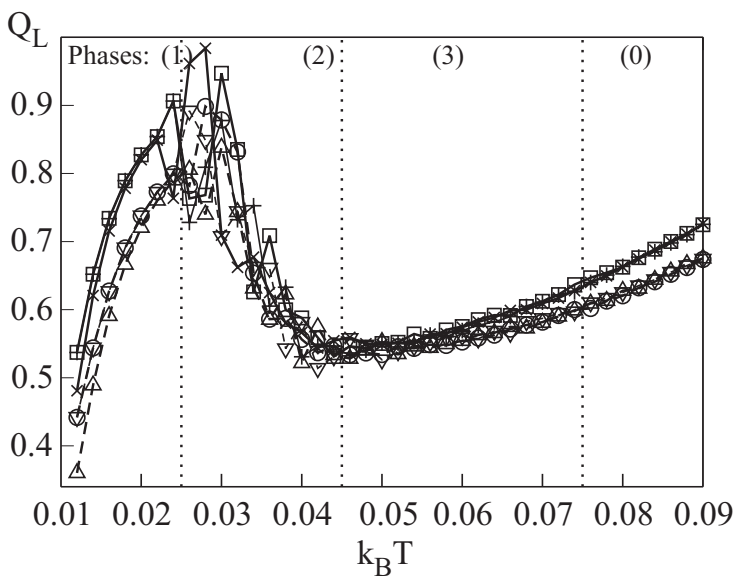

Fig. 5. The $k_{B} T$ dependence of three Binder cumulants $Q$ calculated for the samples with different linear size $L$, listed in the legend box, at $h=0.5$. Each cumulant $Q$ was calculated from the one of three sublattices shown in Fig. 1,c. Phase (1) is absent here. $L=39$, sublattice A $(\nabla) ; L=39$, sublattice B $(\triangle)$; $L=39$, sublattice C (O); $L=51$, sublattice A $(\square) ; L=51$, sublattice $\mathrm{B}(\times) ; L=51$, sublattice $\mathrm{C}(+)$. 
It is worth noting that for $k_{B} T=0$ the ground state boundaries between phases calculated theoretically by Wojtkiewicz [8] are used, respectively $h=0.512,0.584$, 0.888 and 0.928 (see Fig. 3). They very well complement the boudaries obtained here by the Monte Carlo simulations. Thus we may conclude that the ground state boundaries calculated by Datta et al. [4] of $h=0.316,0.352$, 0.384 and 0.404 , respectively, are hardly confirmed.

\section{Conclusions}

This paper presents our MC results which allowed us to establish the finite-temperature phase diagram of the Falicov-Kimball model on the square lattice in the perturbative regime. Although these results in part concerning the phases (3) and (4) are based on indirect conclusions, our analysis evidently proves the existence of stripe ordering also at finite-temperatures in the FKM. The earlier papers showed their existence at low $T$ but did not tell anything about the value of the critical temperature(s). As far as we know, the only exception is the Néel phase [2], the simplest case of our analysis.

Figure 3 is a sketch of the phase diagram and its more systematic MC study will be continued. In particular, we want to explore the dependence of $T_{c}$ 's for the fourth-order phases as a function of $t / U$.

The authors wish to thank Professor R. Lemański for valuable discussions, the Poznań Supercomputing and Networking Center for the access to the supercomputers, and the State Committee for Scientific Research for partial financial support within the grants 4 T11F 01424 (GM) and MERG-CT-2004 No 6348 (LD).

1. L.M. Falicov and J.C. Kimball, Phys. Rev. Lett. 22, 997 (1969).

2. J. Freericks and V. Zlatić, Rev. Mod. Phys. 75, 1333 (2003).

3. J.K. Freericks, E.H. Lieb, and D. Ueltschi, Commun. Math. Phys. 227, 243 (2002).

4. N. Datta, R. Fernandez, and J. Fröhlich, J. Status Phys. 96, 545 (1999).

5. R. Lenański and J. Wojtkiewicz, Phys. Status Solidi B236, 408 (2003).

6. M. Maśka and K. Czajka, Phys. Status Solidi B242, 479 (2005); cond-mat /0504533.

7. G. Musiał, Phys. Rev. B69, 024407 (2004).

8. J. Wojtkiewicz, J. Stat. Phys. 112, 1049 (2003). 